\title{
FACT - Performance of the First Cherenkov Telescope Observing with SiPMs
}

\author{
M. Noethe ${ }^{* b}$, J. Adam ${ }^{b}$, M. L. Ahnen ${ }^{a}$, D. Baack ${ }^{b}$, M. Balbo ${ }^{c}$, A. Biland ${ }^{a}$, M. Blank ${ }^{d}$, \\ T. Bretz ${ }^{a}, \mathbf{K}$. Bruegge $^{b}$, J. Buss ${ }^{b}$, A. Dmytriiev ${ }^{c}$, D. Dorner ${ }^{d}$, S. Einecke ${ }^{b}$, \\ D. Elsaesser ${ }^{b}$, C. Hempfling ${ }^{d}$, T. Herbst ${ }^{d}$, D. Hildebrand ${ }^{a}$, L. Kortmann ${ }^{b}$, \\ L. Linhoff ${ }^{b}$, M. Mahlke ${ }^{a}$, K. Mannheim $^{d}$, S. A. Mueller ${ }^{a}$, D. Neise ${ }^{a}$, A. Neronov ${ }^{c}$, \\ J. Oberkirch ${ }^{b}$, A. Paravac ${ }^{d}$, F. Pauss ${ }^{a}$, W. Rhode ${ }^{b}$, B. Schleicher ${ }^{d}$, F. Schulz ${ }^{b}$,

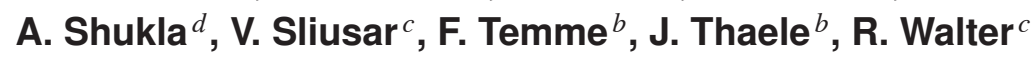 \\ Email: maximilian.noethe@tu-dortmund.de \\ ${ }^{a}$ ETH Zurich, Institute for Particle Physics \\ Otto-Stern-Weg 5, 8093 Zurich, Switzerland \\ ${ }^{b}$ TU Dortmund, Experimental Physics 5 \\ Otto-Hahn-Str. 4, 44221 Dortmund, Germany \\ ${ }^{c}$ University of Geneva, ISDC Data Center for Astrophysics \\ Chemin d'Ecogia 16, 1290 Versoix, Switzerland \\ ${ }^{d}$ Universität Würzburg, Institute for Theoretical Physics and Astrophysics \\ Emil-Fischer-Str. 31, 97074 Würzburg, Germany
}

The First G-APD Cherenkov Telescope (FACT) is pioneering the usage of silicon photo multipliers (SIPMs also known as G-APDs) for the imaging atmospheric Cherenkov technique. It is located at the Observatorio Roque de los Muchachos on the Canary island of La Palma. Since first light in October 2011, it is monitoring bright TeV blazars in the northern sky. By now, FACT is the only imaging atmospheric Cherenkov telescope operating with SIPMs on a nightly basis. Over the course of the last five years, FACT has been demonstrating their reliability and excellent performance. Moreover, their robustness allowed for an increase of the duty cycle including nights with strong moon light without the need for UV-filters.

In this contribution, we will present the performance of the first Cherenkov telescope using solid state photo sensors, which was determined in analysis of data from Crab Nebula, the so called standard candle in gamma-ray astronomy. The presented analysis chain utilizes modern data mining methods and unfolding techniques to obtain the energy spectrum of this source. The characteristical results of such an analysis will be reported providing, e. g., the angular and energy resolution of FACT, as well as, the energy spectrum of the Crab Nebula. Furthermore, these results are discussed in the context of the performance of coexisting Cherenkov telescopes.

35th International Cosmic Ray Conference - ICRC2017

10-20 July, 2017

Bexco, Busan, Korea

${ }^{*}$ Speaker. 


\section{Introduction}

FACT (First G-APD Cherenkov Telescope) [1] is the first Imaging Air Cherenkov Telescope which uses Silicon Photomultipliers (SIPMs) as photo sensors. Observing since first light in October 2011 at the Observatorio del Roque de los Muchachos on the Canary Island of La Palma (Spain), the main goal of FACT is the monitoring of very high energy gamma-ray sources in the Northern hemisphere.

FACT is using a segmented mirror that is comprising 30 hexagonal facets with a total mirror area of $9.51 \mathrm{~m}^{2}$ and a focal length of $4.9 \mathrm{~m}$. The facets are oriented in a hybrid between a parabolic and a Davies-Cotton-geometry [2].

The camera consists of 1440 pixels and has a diameter of approximately $40 \mathrm{~cm}$, resulting in a field of view of $4.5^{\circ}$. Each pixel consists of a solid light concentrator and a SIPM (Hamamatsu MPPC S10362-33-50C) hosting 3600 Geiger-Mode Avalanche Photodiodes (G-APDs). SIPM photo sensors are mechanically and electronically robust, capable of single photon counting, have a low operational voltage, a comparable photon detection efficiency to conventional PMTs and cheap production costs. These promising properties make them particularly suitable for applications in the ground-based Cherenkov gamma-ray astronomy. More details concerning the sensor performance of these photo detectors are given in [3].

The analysis chain starts with the preprocessing of the raw data. For this task, the data analysis tool FACT-Tools $[4,5]$ has been developed. It performs the calibration of raw data, the extraction of the registered Cherenkov photon pulses, selection of pixels likely containing signal, and parameterization of the resulting air shower images. FACT-Tools is an extension of the streams-framework [6] which has been developed in cooperation with the department of computer science at the TU Dortmund.

The image parameters calculated by FACT-TOOls are used to perform an energy estimation of the primary particle and a suppression of background events induced by charged cosmic rays. Both of these tasks are solved using the scikit-learn [7] machine learning framework.

To estimate the main performance characteristics of FACT, observations of the Crab Nebula are used. The Crab Nebula is a nearby Pulsar Wind Nebula and is the brightest source of very high energy gamma-rays, of which no variability has yet been observed, which is making it the so called "standard candle" of gamma-ray astronomy. Several other IACTs have also evaluated their performance characteristics using this source $[8,9]$.

In order to obtain the differential energy spectrum of the Crab Nebula, the resulting data set is unfolded using the software TRUEE [10].

The results in this contribution were achieved by the FACT-ToOls analysis chain, one of the two independent analysis chains used in FACT, the other being MARS [11].

\section{Data Sample}

The observations of the Crab Nebula used to estimate the performance characteristics of FACT were taken between October 2013 and February 2014. A selection was performed, assuring the final sample contains only data taken with a zenith angle below $30^{\circ}$, no clouds, no moon light and a stable trigger rate. This selection results in a total observation time of $t_{\mathrm{obs}}=91.1 \mathrm{~h}$. Observations were 
done in the so-called wobble mode, i. e. not pointing directly at the source, but using a fixed offset of $0.6^{\circ}$ from the camera center, which allows to estimate the background rate at the same time.

Dedicated Monte Carlo simulations are necessary for the analysis of these observations. The gamma-ray and hadron induced extensive air showers are simulated using the software CORSIKA [12]. The produced Cherenkov photons are then used to calculate the telescope response using CERES [11]. The simulated event sets include 12 million air showers induced by gamma-rays in an energy regime between $200 \mathrm{GeV}$ and $50 \mathrm{TeV}$ and about 780 million air showers induced by protons in an energy range between $100 \mathrm{GeV}$ and $200 \mathrm{TeV}$. Both energy spectra follow a power law distribution with an index of -2.7 .

\section{Preprocessing and Image Parameterization}

Preprocessing of both observed and simulated events is done using FACT-ToOls, version $\mathrm{v} 0.17 .2$. In a first step, the raw time series of each pixel are calibrated for the effects of the sampling hardware. After this, the number and mean arrival time of the photons in each pixel are extracted and pixels likely containing no significant Cherenkov light contribution are discarded. The remaining pixels are used for the parameterization of the event. This includes the classical Hillas parameters [13], descriptive statistics of the number of photon and arrival time distributions, and other features describing the shower morphology like the number of distinct groups of pixels after the discarding of non-signal pixels.

\section{Background Suppression}

As cosmic ray induced air showers far outnumber the gamma-ray induced air showers, methods of supervised machine learning are applied to suppress these background events. A random forest classifier [14] trained on 120000 simulated events per class is used. Comprised of 200 decision trees using 22 attributes in total, 4 randomly drawn features at each node and a maximum depth of 15 , an area under the ROC curve of $0.875 \pm 0.003$ is reached. The uncertainty is estimated by a 20 -fold cross validation. The result of applying this classifier is the gamma_prediction $\in[0,1]$, where 1 means that the event is very likely a gamma-ray primary and 0 means the event is very likely induced by charged cosmic rays.

Two different sets of selection cuts are performed for the two tasks of source detection and spectrum estimation. For the source detection, a larger precision ${ }^{1}$ is chosen and for the unfolding more weight is laid on gamma-ray recall ${ }^{2}$.

The chosen cuts are $\theta^{2} \leq 0.1 \mathrm{deg}^{2}$ and gamma_prediction $\geq 0.7$ for the unfolding and $\theta^{2} \leq$ $0.03 \mathrm{deg}^{2}$ and gamma_prediction $\geq 0.85$ for the source detection, where $\theta$ is the angular distance between reconstructed and true source position. Applying these selection cuts yields the effective collection area $A_{\text {eff }}$ as shown in Figure 1.

\footnotetext{
${ }^{1}$ Also commonly refered to as purity

${ }^{2}$ Also commonly refered to as efficiency
} 


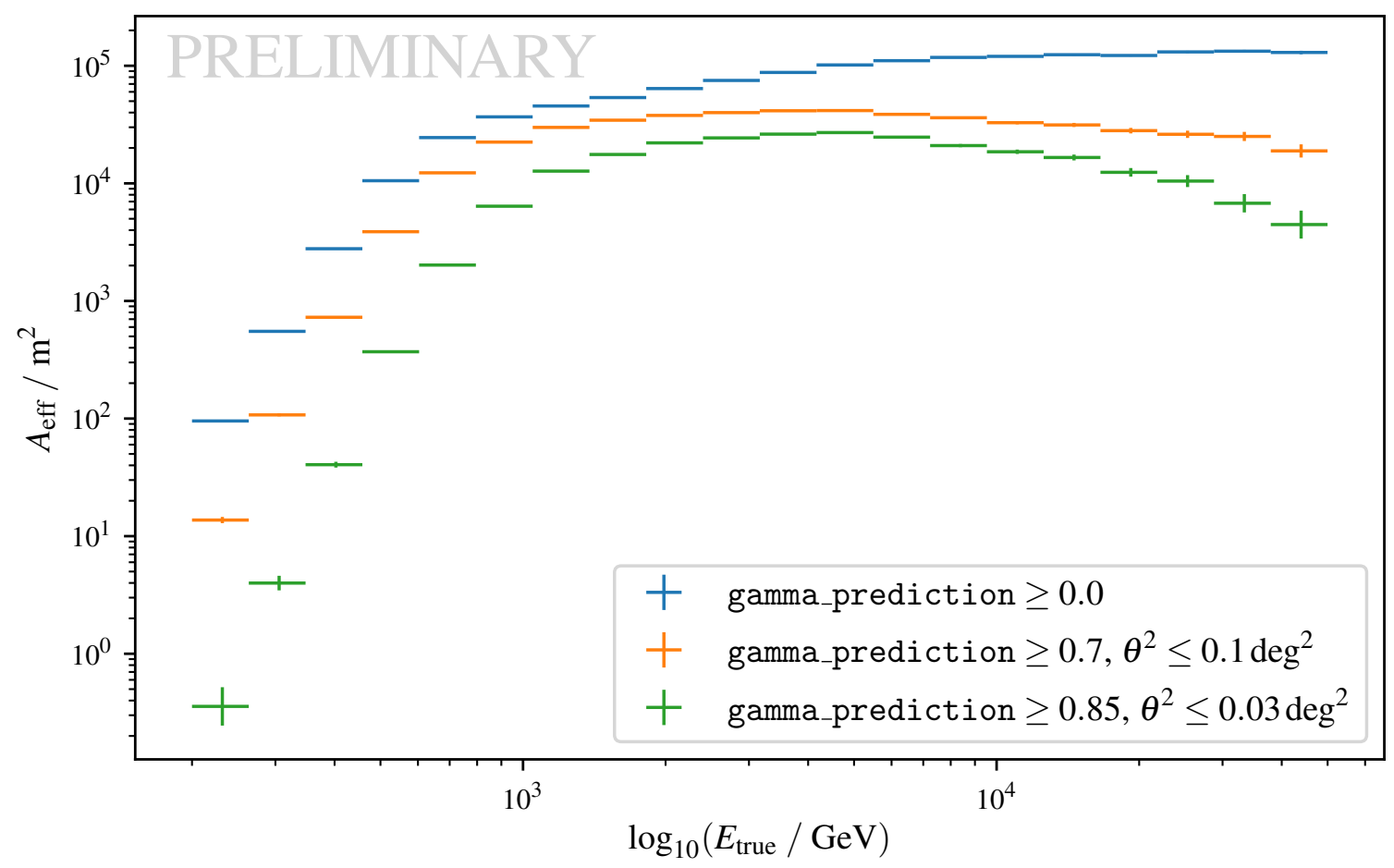

Figure 1: Effective collection area for no selection cuts applied (blue), the unfolding selection cuts applied (orange) and the source detection cuts applied (green).

\section{Energy Estimation}

The energy estimation is also performed using supervised machine learning methods. A random forest regressor trained on 120000 gamma-ray induced events, using 22 attributes, 200 decision trees and 5 random attributes per node is used.

The performance of this energy regressor is estimated using an independent set of simulated gamma-ray events, comparing the estimated energy $E_{\text {est }}$ to the true energy $E_{\text {true }}$.

The complete migration matrix is shown in Figure 2a. Bias and resolution of the estimator are evaluated for bins in $E_{\text {true }}$ as mean and half distance between the $1 \sigma$-quantiles of the relative error

$$
\Delta_{\text {rel }}=\frac{E_{\text {est }}-E_{\text {true }}}{E_{\text {true }}} .
$$

The inter-quantile distance was chosen, because the shape of the $\Delta_{\text {rel }}$ distribution is highly nongaussian, especially in lower energy bins which forbids the common approach of fitting a gaussian and taking its standard deviation as resolution. 


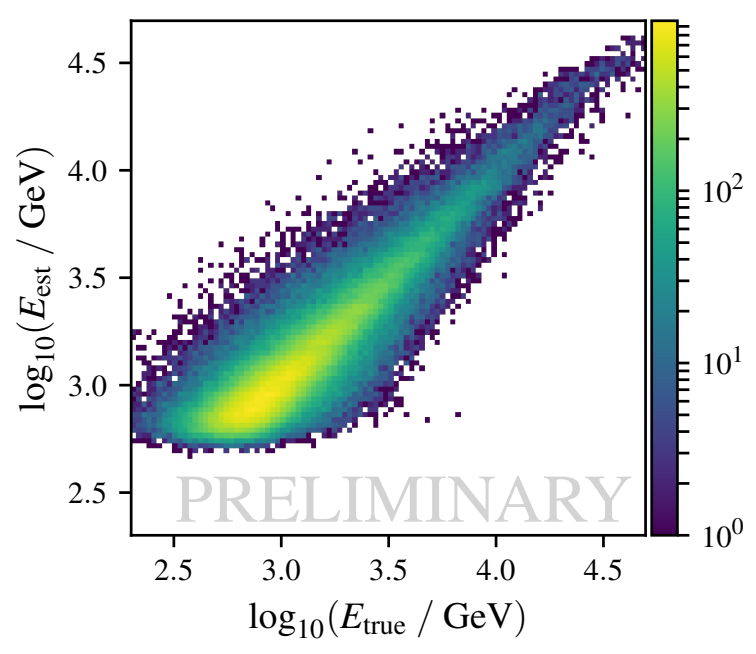

(a) Energy migration.

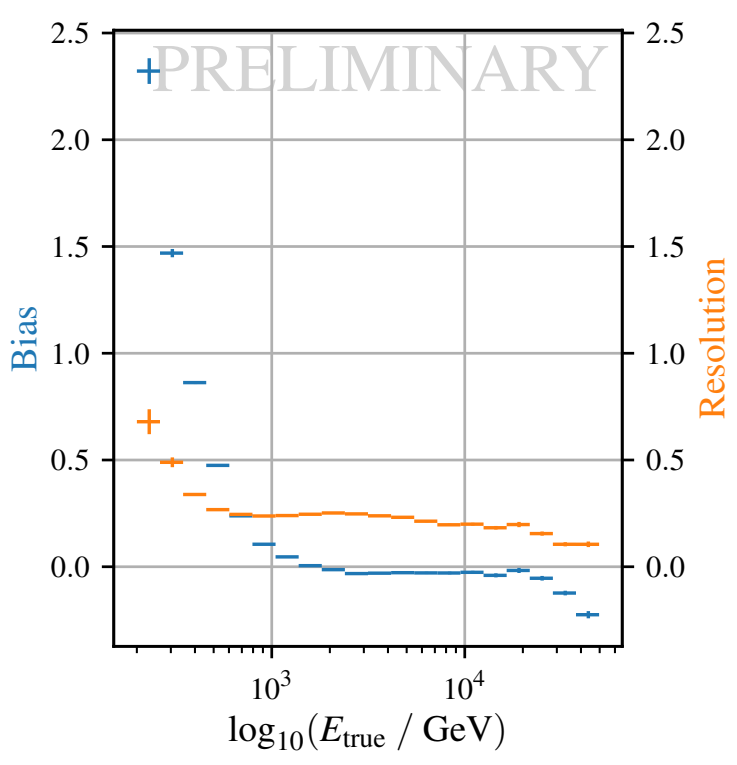

(b) Bias and resolution.

Figure 2: Figure a shows the energy migration. Figure b shows the bias of the energy estimation, calculated as the mean relative error, and the resolution, calculated as half distance between the $1 \sigma$-quantiles of the relative error. Only results for events with gamma_prediction $\geq 0.7$ are shown.

\section{Performance Estimation Using Crab Nebula Data}

The resulting event numbers with respect to the applied event selection cuts and the corresponding detection significances are shown in Table 1. Figure 3 shows the the distance between reconstructed and true source position $\theta$ for the Crab Nebula (On) and the five chosen background regions (Off).

Using TRUEE, the differential flux of the Crab Nebula is obtained. The result is shown in Figure 4. The obtained results are in agreement with results previously obtained by other telescopes.

To estimate the detection potential of FACT, the flux relative to the Crab Nebula that yields $5 \sigma \mathrm{Li} \&$ Ma significance [15] in $50 \mathrm{~h}$ of observation time is calculated. The resulting equation

$$
S_{\text {Li\&Ma }}\left(N_{\text {exc }} \cdot \phi_{\text {rel }}+N_{\text {off }}, N_{\text {off }}, \alpha\right)=5
$$

is solved numerically for the relative flux $\phi_{\text {rel }}$. The uncertainties are estimated by drawing

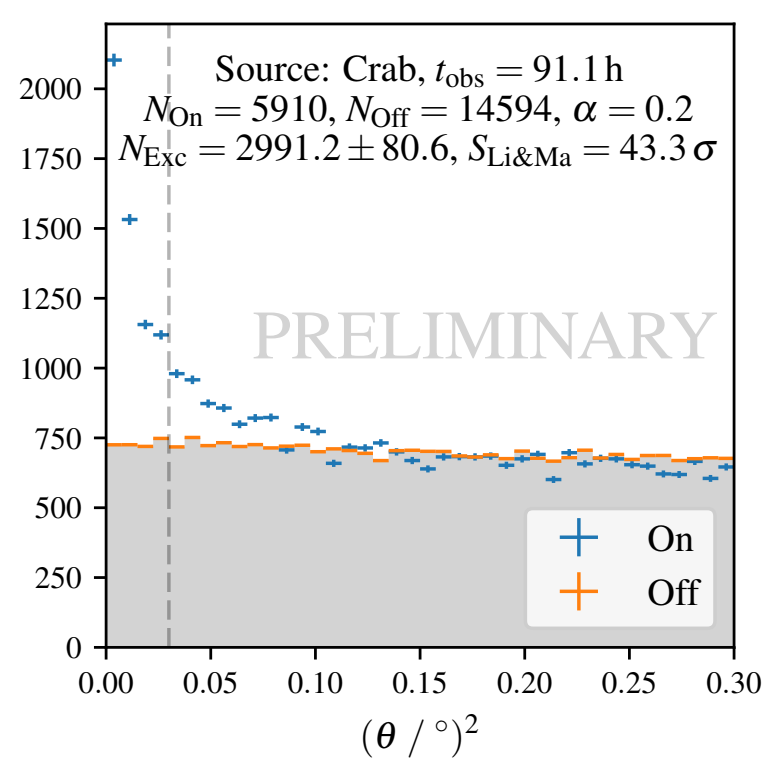

Figure 3: $\theta^{2}$ for the Crab Nebula (On) and the region used for background estimation (Off). A clear excess is visible for the On region and the Crab Nebula is detected with a significance of $43.3 \sigma$ using the Li \& Ma likelihood-ratio test. 
$N_{\text {On }}$ and $N_{\text {Off }}$ from a Poisson distribution and recalculating the sensitivities a 1000 times. Over the whole energy range, an integral sensitivity of $(0.137 \pm 0.004) \mathrm{CU}$ for the source detection cuts and $(0.214 \pm 0.008) \mathrm{CU}$ for the unfolding cuts is achieved.

Table 1: Selection cuts and resulting $N_{\text {On }}, N_{\text {Off }}$, number of excess events $N_{\text {exc }}$ and the resulting significance according to the Li \& Ma likelihood-ratio test.

\begin{tabular}{ccrrcc}
\hline gamma_prediction $_{\text {cut }}$ & $\theta_{\text {cut }}^{2} / \mathrm{deg}^{2}$ & \multicolumn{1}{c}{$N_{\text {On }}$} & \multicolumn{1}{c}{$N_{\text {Off }}$} & $N_{\text {exc }}$ & $S_{\text {Li\&Ma }} / \sigma$ \\
\hline 0.70 & 0.10 & 33735 & 139553 & $5824.4 \pm 198.13$ & 30.6 \\
0.85 & 0.03 & 5910 & 14594 & $2991.2 \pm 80.16$ & 43.3 \\
\hline
\end{tabular}

The differential sensitivity for the nine energy bins used for the spectrum estimation is shown in Figure 5.

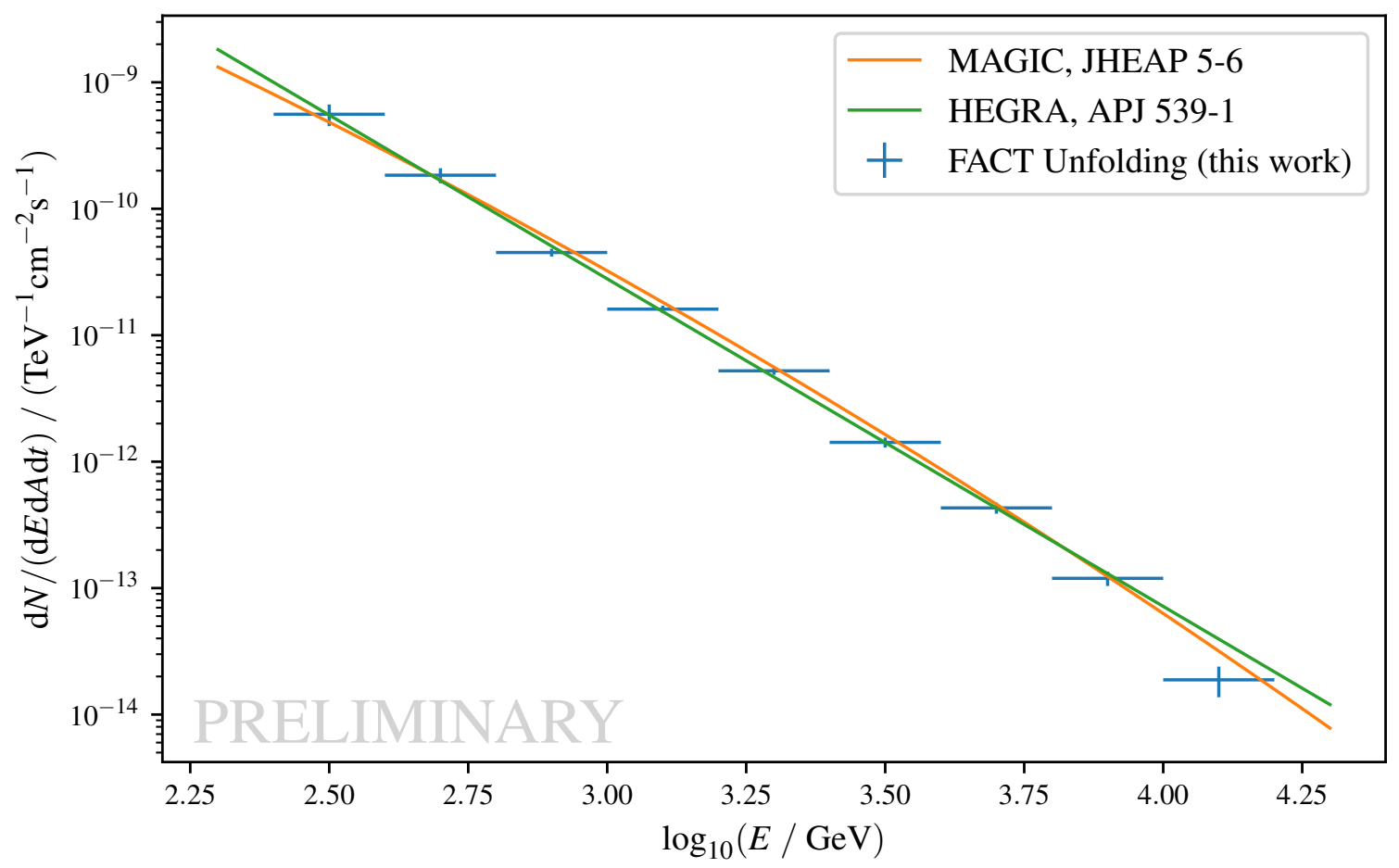

Figure 4: Differential flux of the Crab Nebula as obtained by the TRUEE unfolding. For comparison, the fit results of the MAGIC [16] and the HEGRA [17] experiments are shown as well. 


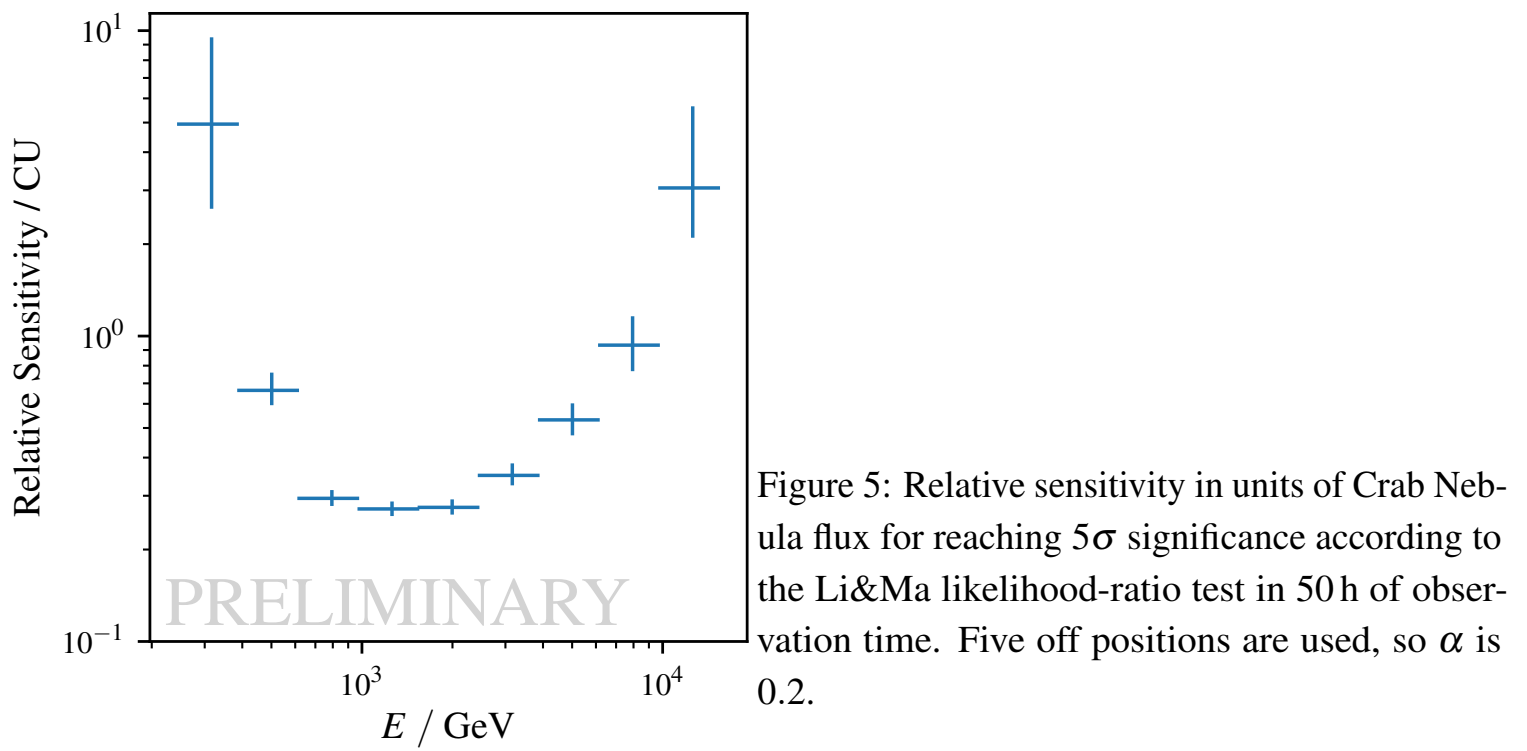

\section{Conclusions}

In this work the analysis chain of FACT including modern machine learning approaches for background suppression and energy estimation has been presented. For the first time, the sensitivity of a SIPM Cherenkov telescope has been evaluated on observations of the Crab Nebula. The source has been detected with a significance of $43.3 \sigma$ in $91.1 \mathrm{~h}$ and the unfolded energy spectrum between $250 \mathrm{GeV}$ and $16 \mathrm{TeV}$ is consistent with measurements of other experiments. With the current analysis methods, FACT reaches an integral sensitivity of $(0.137 \pm 0.004) \mathrm{CU}$. In the near future, it is planned to extend the use of machine learning methods to the reconstruction of the shower origin to improve the angular resolution.

Acknowledgment The important contributions from ETH Zurich grants ETH-10.08-2 and ETH-27.12-1 as well as the funding by the Swiss SNF and the German BMBF (Verbundforschung Astro- und Astroteilchenphysik) and HAP (Helmoltz Alliance for Astroparticle Physics) are gratefully acknowledged. Part of this work is supported by Deutsche Forschungsgemeinschaft (DFG) within the Collaborative Research Center SFB 876 "Providing Information by Resource-Constrained Analysis", project C3. We are thankful for the very valuable contributions from E. Lorenz, D. Renker and G. Viertel during the early phase of the project. We thank the Instituto de Astrofísica de Canarias for allowing us to operate the telescope at the Observatorio del Roque de los Muchachos in La Palma, the Max-Planck-Institut für Physik for providing us with the mount of the former HEGRA CT3 telescope, and the MAGIC collaboration for their support

\section{References}

[1] H. Anderhub et al. "Design and operation of FACT - the first G-APD Cherenkov telescope". In: JINST 8.06 (2013), P06008.

[2] M. Ahnen et al. "Bokeh mirror alignment for Cherenkov telescopes". In: Astroparticle Physics 82 (2016), pp. 1-9. 
[3] A. Biland et al. "Calibration and performance of the photon sensor response of FACT - the first G-APD Cherenkov telescope”. In: JINST 9.10 (2014), P10012.

[4] C. Bockermann et al. "Online Analysis of High-Volume Data Streams in Astroparticle Physics". In: Machine Learning: ECML 2015, Industrial Track. Springer Berlin Heidelberg, 2015.

[5] M. L. Ahnen et al. FACT-Tools GitHub-Repository. URL: https: / / github.com/factproject/fact-tools.

[6] C. Bockermann. "Mining Big Data Streams for Multiple Concepts". PhD thesis. 2015.

[7] F. Pedregosa et al. "Scikit-learn: Machine Learning in Python". In: Journal of Machine Learning Research 12 (2011), pp. 2825-2830.

[8] J. Aleksić et al. "The major upgrade of the MAGIC telescopes, Part II: A performance study using observations of the Crab Nebula". In: Astroparticle Physics 72 (2016), pp. 76-94.

[9] J. Holder et al. "Status of the VERITAS Observatory". In: AIP Conference Proceedings. Vol. 1085. 1. AIP. 2008, pp. 657-660.

[10] N. Milke et al. "Solving inverse problems with the unfolding program TRUEE: Examples in astroparticle physics". In: NIM A 697.0 (2013), pp. 133-147.

[11] T. Bretz and D. Dorner. "MARS - CheObs goes Monte Carlo". In: Proceedings of the 31st ICRC. 2009.

[12] D. Heck et al. CORSIKA: A Monte Carlo Code to Simulate Extensive Air Showers," Report FZKA 6019, Forschungszentrum Karlsruhe. Tech. rep. 1998.

[13] A. M. Hillas. "Cerenkov light images of EAS produced by primary gamma". In: Proceedings of the 19th International Cosmic Ray Conference. Vol. 3. 1985, pp. 445-448.

[14] L. Breiman. "Random Forests". English. In: Machine Learning 45.1 (2001), pp. 5-32. ISSN: 0885-6125. DOI: $10.1023 / \mathrm{A}: 1010933404324$.

[15] T.-P. Li and Y.-Q. Ma. "Analysis methods for results in gamma-ray astronomy". In: The Astrophysical Journal 272 (1983), pp. 317-324.

[16] J. Aleksić et al. "Measurement of the Crab Nebula spectrum over three decades in energy with the MAGIC telescopes". In: Journal of High Energy Astrophysics 5 (2015), pp. 30-38.

[17] F. Aharonian et al. "The energy spectrum of $\mathrm{TeV}$ gamma rays from the Crab Nebula as measured by the HEGRA system of imaging air cerenkov telescopes". In: The Astrophysical Journal 539.1 (2000), p. 317. 